\title{
Frontières
}

\section{Mourir dans la dignité : un défi pour l'humain, un lieu pour Dieu ?}

\section{Dominique Jacquemin}

Volume 20, numéro 1, automne 2007

La « bonne mort »

URI : https://id.erudit.org/iderudit/017942ar

DOI : https://doi.org/10.7202/017942ar

Aller au sommaire du numéro

\section{Éditeur(s)}

Université du Québec à Montréal

ISSN

1180-3479 (imprimé)

1916-0976 (numérique)

Découvrir la revue

Citer cet article

Jacquemin, D. (2007). Mourir dans la dignité : un défi pour l'humain, un lieu pour Dieu ? Frontières, 20(1), 12-21. https://doi.org/10.7202/017942ar
Résumé de l'article

Prenant appui sur l'expression très médiatisée en France de « mourir dans la dignité ", l'auteur se demande comment l'expérience de la mort peut de nos jours constituer un lieu conjoint de la dignité et de l'homme et de Dieu. Sans renoncer à une approche ontologique de la dignité telle qu'elle se dessine dans la Déclaration des droits de l'homme de 1948, il plaide davantage pour une approche clinique de cette dernière : c'est au niveau de l'expérience concrète d'être respecté et accompagné comme humain que la personne malade appréhende qu'il existe un au-delà de l'altération capable de la maintenir dans sa dignité d'humain. C'est ce même comportement qui atteste conjointement de la dignité de Dieu qui, dans sa kénose, dit sa capacité à ne pas être étranger de l'aventure humaine. 


\section{Résumé}

Prenant appui sur l'expression très médiatisée en France de «mourir dans la dignité ", l'auteur se demande comment l'expérience de la mort peut de nos jours constituer un lieu conjoint de la dignité et de l'homme et de Dieu. Sans renoncer à une approche ontologique de la dignité telle qu'elle se dessine dans la Déclaration des droits de l'homme de 1948, il plaide davantage pour une approche clinique de cette dernière: c'est au niveau de l'expérience concrète d'être respecté et accompagné comme humain que la personne malade appréhende qu'il existe un au-delà de l'altération capable de la maintenir dans sa dignité d'humain. C'est ce même comportement qui atteste conjointement de la dignité de Dieu qui, dans sa kénose, dit sa capacité à ne pas être étranger de l'aventure humaine.

Mots clés: dignité - mort -

accompagnement -

éthique et théologie du soin.

\section{Abstract}

Adopting as a corner-stone the expression "dying with dignity" which is widespread in the media in France, the author wonders how the experience of death can constitute today a place of convergence for the dignity both of man and God. Although he does not abandon the ontological approach to dignity such as it is described in the Declaration of Human Rights of 1948, he rather favours a clinical approach to it: it is through the concrete experience of being respected and accompanied as a human being that the patient grasps that something beyond ailment exists which is capable of maintaining him in a state of dignity worthy of a human being. It is this same behaviour that also testifies to the dignity of God who, in his kenosis, expresses his capacity not to remain an outsider to the human adventure.

Keywords: dignity - death accompanying - ethics and theology of caring.

\section{Mourir dans la dignité Un défi pour l'humain, un lieu pour Dieu?}

\section{Dominique Jacquemin, \\ enseignant-chercheur, Université catholique de Lille, responsable d'équipe d'aumônerie, \\ Cliniques universitaires UCL de Mont-Godinne.}

Mourir dans la dignité: un défi pour l'humain, un lieu pour Dieu? Voilà un intitulé apparemment simple, concis dont nous voudrions laisser résonner tous les termes dans leur complexité, y compris le point d'interrogation. En effet, si le fait de mourir est une des expériences les plus naturelles qui soient, coextensive à notre naissance, il n'est pas évident qu'elle soit appréhendée et vécue de la sorte par l'homme contemporain immergé dans un univers médico-technique lui offrant l'illusion d'une vie sans fin, modelé qu'il est socialement et culturellement par une représentation de la vie, du corps et de l'existence qui s'assimilerait volontiers à un bonheur traduisible par l'absence de toute limite. Dans ce contexte, que peut signifier «mourir dans la dignité» lorsque cette revendication associative a fait de la dignité même un concept composite, comme si la notion de dignité dans «le sens absolu pouvait se confondre avec un sens plus relatif» (Echard, 2006, p. 79), comme s'il était possible d'opposer dignité et indignité, dignité et perte de dignité en termes d'altération du corps, d'incapacité d'action et de négation du sujet autonome?

Ces quelques éléments nous conduisent aisément à la notion de défi pour l'humain tant, toutes et tous, que nous le voulions ou non, sommes redevables de cet imaginaire social, médical nous conduisant, pour autrui ou pour nous-mêmes, à cette interrogation perplexe, angoissante pour certains: l'expérience de la mort est-elle encore un lieu d'humanité ? Quant à l'interrogation ultime - un lieu pour Dieu? -, elle questionnera davantage le croyant. Si la tradition chrétienne fait de la vie un chemin préparant à la rencontre de Dieu, finalité de l'existence, il faut bien reconnaître que pour l'homme contemporain et, parfois, pour celles et ceux qui l'accompagnent humainement et pastoralement, ce serait vite dit. Qui vit encore cela, comme le souligne T. Radcliffe (Radcliffe, 2005, p. 40), cet exode de l'existence comme aventure humaine et croyante conduisant à la rencontre de Dieu, comme traduction existentielle de l'espérance chrétienne (1Co 2,9)? De plus, lorsque l'homme contemporain, modelé par un rapport à la maîtrise et à l'efficacité, fait l'expérience de la finitude qui vient, il n'aura bien souvent qu'un reproche à formuler - «qu'ai-je fait 
au Bon Dieu?» - comme si l'expérience de la finitude et de la mort qui vient était devenue, quasi par nature, un lieu étranger pour Dieu. Nous sommes donc assez loin de cet idéal qui en ferait un lieu d'accomplissement: «Elle [la mort] est l'expérience vitale ultime, celle qui nous force à tout lâcher, à abandonner notre besoin irrésistible de dominer toute chose » (Évêques de Belgique, 1994, p. 233).

Si nous ne sommes pas extérieurs à ce que nous questionnons - l'expérience de la mort et les représentations contemporaines de la dignité -, il faut reconnaître que le sujet n'est pas simple d'autant que nous ne sommes en rien assurés de nos ressources effectives lorsque nous y serons confrontés, tant il est toujours plus aisé de parler pour l'autre. Cependant, nous pensons que la question peut être non seulement rencontrée mais surtout soutenue dans les pratiques soignantes et pastorales. C'est ce à quoi nous aimerions nous arrêter dans cette contribution qui se développera en trois temps.

Dans un premier temps, nous nous arrêterons à la notion de "défi pour l'homme». En effet, il nous semble essentiel de bien appréhender ce lieu humain qu'est l'expérience du mourir vécu essentiellement dans le contexte de la techno-médecine, non pour la décrier mais pour nous rendre compte que le concept de dignité n'est plus nécessairement un concept spontané lorsque l'humain en son corps, soutenu par la médecine tout au long de son existence, devrait approcher l'expérience de la mort comme si cette même médecine n'avait pas, d'une manière ou d'une autre, modifié l'autocompréhension qu'il a de lui-même, en d'autres mots son anthropologie.

Dans un deuxième temps, rencontrant l'expérience de nombreux soignants et bénévoles, nous essayerons de montrer en quoi et comment un certain exercice de la médecine permet de soutenir, de reconnaître et de nommer en acte cette dignité de la personne humaine faisant l'expérience de la mort qui vient. Si cette visée d'humanité fut particulièrement restaurée par la pratique des soins palliatifs (Jacquemin, 2004), elle nous indique cependant deux choses. Tout d'abord, une médecine technique, spécialisée peut rencontrer autrui souffrant dans sa dignité mais c'est surtout une pratique professionnelle et d'accompagnement qui donnera au concept même sa réalité plutôt qu'une affirmation a priori de son existence. En d'autres mots, modelés comme nous le sommes, ce serait davantage l'expérience du soin comme rencontre humaine qui nous permettrait de ne pas nous expérimenter indignes dans notre humanité souffrante et proche de la mort.

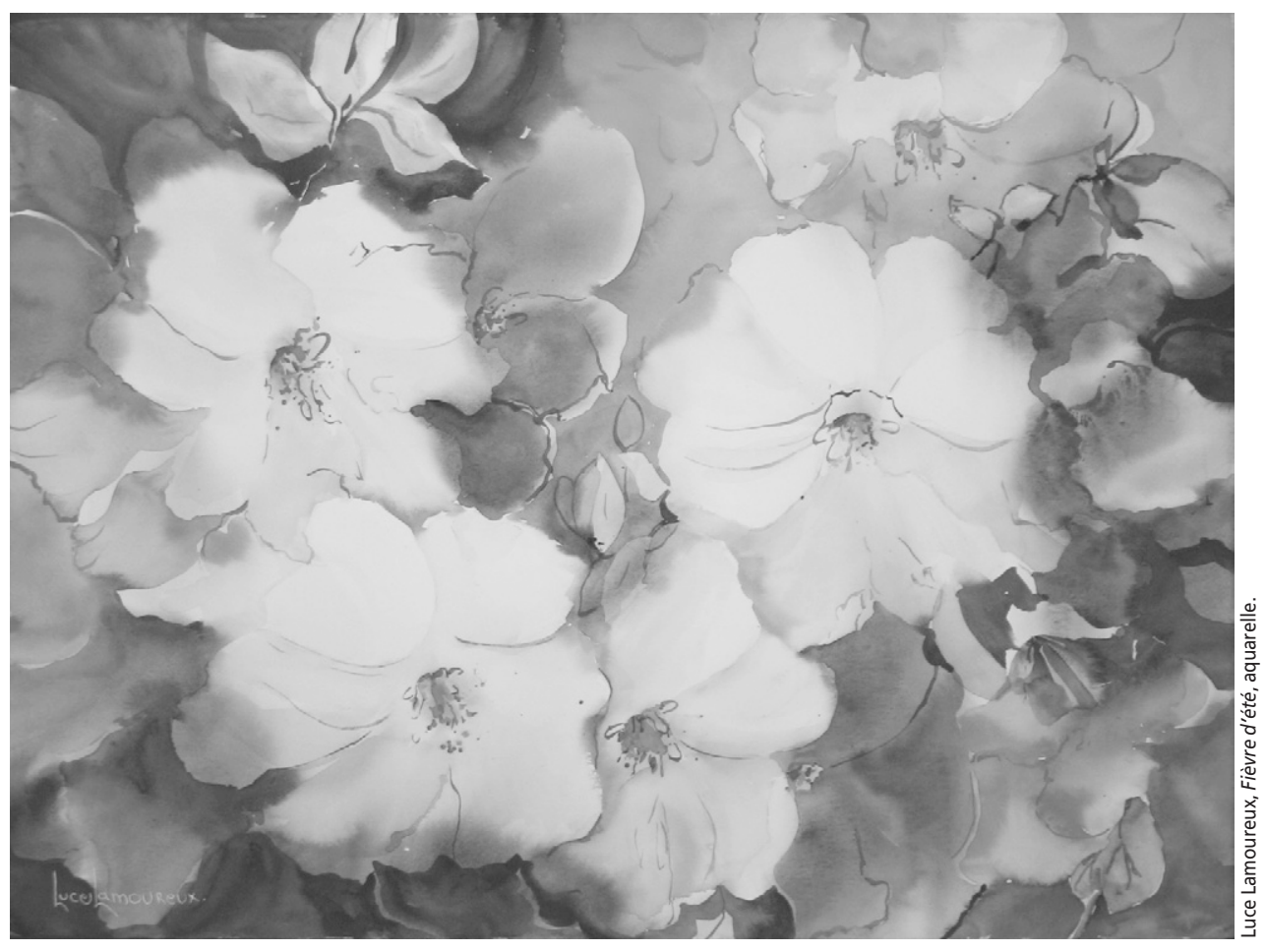

Enfin, dans un troisième temps, nous aimerions considérer le dernier terme de notre interrogation: mourir dans la dignité, un lieu pour Dieu? Il nous semble que cette question mérite au moins trois pistes de réflexion. Il s'agira tout d'abord de se demander si l'expérience de la mort, cette descente au plus bas de soi (Bellet, 2005), cette épreuve vécue par bon nombre de nos contemporains comme un lieu hors sujet, hors humanité, ne peut pas être nommée théologiquement le lieu de l'homme parce qu'il serait le lieu de Dieu. La réponse positive à cette question devrait nous permettre de montrer l'importance de l'accompagnement soignant et pastoral comme lieu théologique, espace de rencontre où se nomme le Dieu auquel nous essayons de croire. S'il devait en être de la sorte, l'engagement humain pourrait être nommé un lieu ecclésial attestant de la sollicitude de Dieu pour le monde, à l'image de ce que dit l'Église d'elle-même dans le concile Vatican $\mathrm{II}^{1}$.

\section{" MOURIR DANS LA DIGNITÉ », UN DÉFI POUR L'HUMAIN}

\section{MOURIR AU CCEUR DE LA MÉDECINE}

S'il nous faut maintenant commencer à réfléchir au concept de dignité, il nous semble essentiel de le réfléchir au cœur du lieu où, concrètement, il se trouve aujourd'hui appréhendé : la médecine. De plus en plus, habitués que nous sommes à être pris en charge par une médecine technique, efficace, gérant tous nos maux, l'homme contemporain en est arrivé à se forger une image de ce que serait sa fin supportée par cette même médecine : une «bonne mort», c'est-à-dire un moment de l'existence sur lequel il pourrait exercer une pleine maîtrise dans une certaine "dimension esthétique », autrement dit sans être dégradé, porteur d'une belle image de soi, autonome, ce terme étant envisagé ici comme "non poids», "non à charge », capable d'indépendance tant physique que psychique; qu'il suffise de penser ici à nos représentations spontanées de la maladie d'Alzheimer. Cette conception de la fin de vie se trouve de plus en plus prégnante avec l'allongement de l'existence humaine amenant un changement important au niveau des mentalités, une sorte de mutation anthropologique (Ameisen et al., 2003, p. 94) : on meurt plus vieux et l'homme contemporain craindra moins la mort en tant que telle - convaincu dans son imaginaire que la médecine peut quasi indéfiniment en postposer la venue - que la perte de son autonomie, l'advenue du temps de la dépendance et de la dégradation, sentiments qui conduiront aisément à des testaments de vie à visée protectrice, à une augmentation des discours et demandes relatives à l'euthanasie comme capacité de «garder sa vie en mains».

De plus en plus, comme l'indique Isabelle Marin (Marin, 1991, p. 97-101), le concept de dignité ne se trouve plus appréhendé qu'entre deux pôles, la notion esthétique et la réalité de la maîtrise, voire de la toute-puissance, et la dignité risque de ne plus être convoquée que comme un concept a priori pour mettre en question d'autres réalités que seraient l'échec et l'efficacité thérapeutique médicale mise à 
mal. Dans un tel contexte, cette notion de dignité conduirait à une impasse puisqu'il serait plus courageux de préférer la mort «dans la dignité »- position de certains membres de l'Association pour le droit de mourir dans la dignité - que d'accepter de se soumettre à la persistance d'une vie devenue indigne. Ce risque d'impasse se trouve d'autant plus important si on $\mathrm{y}$ adjoint nos représentations de la douleur, souvent assimilées indûment à la notion de souffrance, qui nous feraient dire que "c'est pire que la mort» (Marin, 1991, p. 98).

Ces quelques éléments nous permettent déjà de nous rendre compte que, de nos jours, le concept et la réalité de la dignité au cœur de la maladie, de l'expérience souffrante, de la dégradation physique et psychique, du mourir ne seront plus spontanés et solliciteront un engagement éthique, un tiers restaurateur de ce qui en soi n'a jamais été perdu mais est devenu de moins en moins lisible, dicible, attesté: "Le regard et la parole d'autrui font revivre l'homme dans l'homme. Nous sommes loin de la dignité comme image ou maîtrise d'un destin. La dignité se comprend alors dans une relation humaine, produite par la reconnaissance de l'autre»(Marin, 1991, p. 98). Cette conception de la dignité est importante à considérer car elle semble renvoyer à une sorte de pari éthique qui consisterait à pouvoir aller au-delà de la seule image de soi-même ou de l'autre altéré, ce que nous nommerions volontiers ici l'autre comme idole de soi - le point de vue idéalisé que j'aurais de lui-même - ou comme idole de moi - l'autre qui ne pourrait que me renvoyer une image valorisante de moi-même ou porteuse du risque de ma propre altération -, deux situations qui, considérées pour elles-mêmes et sans distance critique, risqueraient d'être qualifiées de nos jours de non-sens. S'il est ici question de parler de dignité en termes d'engagement éthique, ce serait pour souligner la dimension active, engageante que ce concept et cette reconnaissance doivent acquérir comme en amont de toute rencontre clinique pour affirmer que la dignité de la personne en fin de vie est inaliénable, qu'elle représente une visée pratique sollicitant de nos jours l'engagement éthique du personnel soignant et accompagnant. En ce sens, parler de dignité de la mort au cœur de la médecine contemporaine ne pourrait, à nos yeux, être possible que comme une visée toujours à réassumer dans une dynamique interindividuelle et sociale si c'est bien un certain imaginaire social qui risque, de nos jours, d'en offrir une compréhension tronquée.

\section{MORT ET REPRÉSENTATION DU CORPS} COMME LIEU DU BONHEUR

Après avoir envisagé dans les grandes lignes l'importance que peut avoir l'exercice concret de la médecine pour appréhender le concept de dignité, nous aimerions soulever une autre piste de réflexion tout aussi importante: la conception que nous nous faisons du corps, notre corps pour penser notre rapport personnel, individuel à la notion de dignité trop souvent réduite à une non-altération du corps, lieu identifié du bonheur individuel: est heureux celui qui n'est ni malade, ni altéré, autonome au sens où nous le présupposions antérieurement.

Toutefois, il serait simpliste d'assigner la responsabilité à la seule médecine si nous voulons réfléchir le lien entre dignité, société et médecine pour pointer les difficultés que des professionnels peuvent éprouver, au regard de ce lien, dans la pratique soignante. En effet, et certainement en correspondance avec l'émergence d'une capacité opératoire de la médecine, c'est à une nouvelle perception-conception du corps comme lieu du bonheur subjectif que nous sommes aujourd'hui confrontés, réduisant à cette seule dimension subjective individuelle la notion de dignité. Nous aimerions, à ce sujet, reprendre certains éléments que nous en donne Hervé Juvin dans un ouvrage récent où il traite de l'avènement du corps (Juvin, 2005).

Il part d'un constat, celui d'un nouveau statut du corps qui ne pourrait plus être appréhendé comme un poids, une contrainte mais bien comme le lieu du sujet et de son épanouissement: "Pour la première fois, dans l'histoire d'un Occident soumis à l'affrontement du bien et du mal, de l'âme et du corps, de l'éternité et de l'instant, la bonne vie est la vie consacrée au plaisir. Être quelqu'un de bien, ce n'est plus humilier ce corps voué au péché, imposer pénitence à la chair faible, exercer sa conscience et s'exercer par la prière à quitter cette vie ici-bas, c'est bien vivre. Malheur à celui qui passe un jour sans se réjouir de soi »(Juvin, 2005, p. 58). Ces quelques éléments nous indiquent que c'est bien le corps qui est devenu le destinataire et le lieu de la vie bonne, le lieu du sujet au détriment d'un certain rapport au monde et au temps. H. Juvin va tirer de ce constat certaines conséquences intéressantes pour mieux appréhender la question de la souffrance et de la volonté du sujet. En effet, le rapport à la souffrance en son corps devient le lieu de la volonté du sujet: elle n'est possible que pour autant que le sujet $\mathrm{y}$ concède - par exemple la souffrance d'un sport extrême - et le corps se devra d'être ce que le sujet veut qu'il soit, porteur de santé, d'épanouissement, de bien-être, de bonheur. Le corps, s'il est le lieu de l'identité du sujet, devra correspondre aux visées du sujet individuel: "la conquête de la mort agie, de la mort choisie, de la mort volontaire est l'ultime étape de l'invention d'un corps neuf » (Juvin, 2005, p. 80). Dans cette dynamique, on assistera assez rapidement à une équivalence entre altération du corps et altération-négation du sujet, vie de malheur, existence indigne.

DE NOS JOURS, LE CONCEPT ET LA RÉALITÉ DE LA DIGNITÉ AU CFUR DE LA MALADIE, DE L'EXPÉRIENCE SOUFFRANTE, DE LA DÉGRADATION PHYSIQUE ET PSYCHIQUE, DU MOURIR NE SERONT PLUS SPONTANÉS ET SOLLICITERONT

\section{UN ENGAGEMENT ÉTHIQUE.}

Cette approche ne sera pas sans conséquences pour la médecine et ce qu'on peut en espérer en termes de dignité. Elle se devra de soutenir le sujet dans son rapport au corps construit, c'est-à-dire relu comme «celui » qui porte mon bonheur, tant dans une appréciation personnelle que dans le regard des autres: "je me sens bien et les autres me voient bien» (pensons ici à nos regards spontanés sur la personne altérée, handicapée). Il s'en suivra également, socialement et de plus en plus au cœur de la médecine, un accroissement de la responsabilité du sujet à l'égard de son propre corps: «Avec la capacité de le produire, la responsabilité de chacun à l'égard de son corps, de sa séduction, de sa performance introduit de nouvelles catégories du bien et du mal - chacun est responsable de son corps» (Juvin, 2005, p. 150). Corps valorisé, idéalisé, de plus en plus protégé par un ensemble législatif, il deviendra souvent, grâce à la médiation de la médecine, l'objet d'une requête sociale de plus en plus forte, au risque cependant de désapproprier le sujet de sa propre subjectivité ou de lui imposer une excessive responsabilité. Peut-être serait-il possible, dans ce même état d'esprit, d'appréhender les corpus législatifs qui se développent actuellement dans la visée, certes légitime mais ambiguë, de protéger le sujet dans l'individualité de son propre rapport au corps $^{2}$. 
Ce déplacement anthropologique est important au regard d'une pratique professionnelle et de l'appréciation individuelle et collective de la notion de dignité. En effet, quel est ce corps, porteur de quelle vision d'un idéal construit, dont le soignant prend soin, surtout si ce corps, en sa dimension charnelle, risque de dire l'adéquation de la personne à sa propre dignité? Faisant le pari que nous sommes à titre personnel plongés dans un imaginaire social, comment cette vision du corps nous traverse-t-elle ou non dans une pratique professionnelle et dans ce que d'autres - patients, familles, société - en attendent? D'une manière plus large, cette approche du corps n'est pas sans qualifier la pratique des soins palliatifs dans leur mandat de soutien et de restauration de la dignité des plus fragiles. En effet, même si ce lien sujet-corps peut poser question lorsqu'il est mis en adéquation avec la réalité de la personne, le soin du corps prendrait ici encore davantage d'importance en atteignant le sujet dans ce qui serait devenu son identité, c'est-à-dire son corps (Jacquemin, 2004, p. 76-84). Mais il est un revers à la médaille: l'altération du corps singulier deviendrait peut-être un obstacle à la solidarité sociale dans la mesure où l'individu pensé en son corps comme lieu du bien-être, du bonheur deviendrait la seule motivation de l'engagement et de l'action bonne.

\section{QU'EN EST-IL DE LA DIGNITÉ?}

Ces premiers points d'appui relatifs à une nouvelle conception qu'aurait le sujet contemporain de lui-même à la suite de son inscription de plus en plus prégnante dans une médecine objective et technique pensée comme lieu du bonheur, pour ne pas dire de salut, doivent-ils nous faire renoncer au concept inaliénable de dignité, surtout si nous semblons en faire ici une attitude clinique relevant d'un défi éthique à assumer quotidiennement au cœur du soin?

Nous ne pouvons que faire nôtre la Déclaration universelle des droits de l'homme de 1948 et la place qu'elle accorde à la dignité dans les droits fondamentaux: "Tous les hommes naissent libres et égaux en dignité et en droits, ils sont doués de raison et de conscience et doivent agir les uns avec les autres dans un esprit de fraternité.» Nous irons même plus loin en faisant nôtre l'approche d'un collectif de médecins catholiques: "La dignité de l'homme est inaliénable et indépendante de son état du moment; elle est inhérente au fait qu'il a été créé à l'image de Dieu. Cette dignité s'inscrit dans une histoire, de la naissance à la mort et au-delà. Dans la maladie, tout homme reste digne; c'est intrinsèque à sa nature »(Collectif, 2000, p. 5).

Non, il ne faut pas renoncer au concept de dignité comme une dimension inaliénable caractérisant l'être humain en bonne santé ou malade. La dignité est et reste au fondement de toute pratique soignante et de la déontologie médicale ${ }^{3}$. Mais, en même temps, il faut reconnaître que, dans le contexte social qui est le nôtre, elle n'apparaît plus comme une dimension spontanée continuant de qualifier l'être humain souffrant, proche de la mort même si, moyennant l'engagement des proches, des soignants, ce même humain continuera à s'expérimenter digne de vivre et de mourir en sa pleine humanité. La nuance pourra peut-être paraître étrange à certains, mais elle ne fait que renvoyer, en toute honnêteté, à nos visions de l'humain, et donc de nous-mêmes, lorsque nous nous trouvons face à l'autre altéré: qui voudrait spontanément être comme lui ? Et le pas est aujourd'hui trop vite franchi pour faire de la dignité un seul qualificatif provisoire de l'existence humaine alors qu'elle dit notre commune condition d'humain. Aussi, ce risque étant connu, nous préférons déployer ici une conception de la dignité de la vie, et donc du mourir, certes toujours présente mais que le malade aurait de nos jours à expérimenter dans les soins pour s'en convaincre tant une certaine anthropologie et une vision sociale auraient tendance à l'obturer dans une perception idéalisée de la vie humaine.

\section{UN ENGAGEMENT PROFESSIONNEL ET PASTORAL COMME ATTESTATION DE LA DIGNITÉ HUMAINE}

Dans ce deuxième temps de l'exposé, nous voudrions indiquer comment la personne souffrante peut expérimenter une dignité de l'affectation et du mourir grâce à l'engagement humain de la médecine et de ses professionnels, attestant de l'engagement promu par Xavier Thévenot: "Parler de dignité, c'est donc militer en faveur d'une éthique faisant pleinement droit à la gratuité, à un respect "sans pourquoi”" (Thévenot, 2000, p. 211). En effet, à travers une certaine manière de poser l'acte d'accompagnement et de soin, l'engagement humain et professionnel «dit» la dignité de l'humain malade et permet à ce dernier de continuer à s'expérimenter dans sa dignité d'humain, de semblable. En d'autres mots, il s'agirait moins ici de décliner ce qu'est la dignité mais de montrer comment elle se dit et est reconnue à travers certaines dimensions éthiques de tout acte de soin (Jacquemin, 2004, p. 21-28), comment l'autre souffrant s'y trouve attesté dans toute sa dignité d'humain.

\section{UNE APPROCHE DU TEMPS RÉINTÉGRÉE}

Une première modalité de reconnaissance de cette dignité résiderait dans la manière de réintégrer le temps dans sa dimension d'histoire et d'histoire individuelle de tout patient. Nous empruntons cette conviction à Bernard Matray lorsqu'il mettait en évidence deux conditions préalables pour la mise en œuvre de soins palliatifs: «[...] accepter le temps du mourir comme un temps qui, marqué d'une particularité propre, n'en est pas moins, lui aussi, une partie intégrante de l'histoire de la personne; il est nécessaire, d'autre part, de tenter, durant ce temps, de "faire société" avec les grands malades et leurs familles» (Matray, 1995, p. 7).

Cette acceptation du temps du mourir comme un temps à vivre demande certes un engagement éthique de tout soignant, tout accompagnant et nécessite conjointement de lever certaines résistances: il est difficile humainement d'être confronté à une personne en fin de vie, ne fût-ce que parce qu'elle nous renvoie l'idée de notre mort proche; c'est l'image de l'autre altéré, souffrant qui nous renvoie à notre propre vulnérabilité; ce sont ses propos, ses plaintes, ses questions parfois difficiles à entendre et à accepter. Cependant, il importe, dans ces derniers temps, de continuer à croire et à manifester que le malade, «même dans la plus grande détresse, [...] est une personne humaine qui doit être aidée et respectée comme toute autre» (Matray, 1995, p. 7). Il ne s'agit certes pas d'idéaliser la fin de la vie - elle reste le lieu d'une crise, parfois sans solution réelle d'accompagnement -, mais bien de continuer à considérer l'autre dans le respect, l'attention, les soins qu'il mérite.

Cette dimension de présence à l'autre dans son histoire, dans le temps ultime qu'il vit encore, où des besoins spécifiques peuvent encore s'exprimer, nécessite une attitude de la part des soignants et accompagnants, attitude que nous réapprennent peut-être les soins palliatifs, celle de «faire société » (Matray, 1995, p. 8) avec le malade, c'est-à-dire d'entrer en sa compagnie dans une certaine solidarité, d'être présent dans ce lieu où lui se trouve en cette période de vie. Cette attitude consiste à croire qu'au-delà de ce qui semble perdu, indigne diraient certains, il y a encore quelque chose à faire même s'il nous faut sortir de ce que l'on considère habituellement le domaine de la médecine - traiter, guérir -, c'est-à-dire d'accepter de maintenir, ou de réintroduire, le temps du mourir dans la communication comprise au sens large (gestes, attitudes, présences, 
paroles, en un mot tout ce qui continue à faire sens). Fondamentalement, cette attitude renvoie dans la foi en cette capacité d'être simplement au côté de l'autre parce qu'il me vaut, "parce qu'il est à l'image de Dieu, l'individu humain a la dignité de personne: il n'est pas simplement quelque chose, mais quelqu'un. Il est capable de se connaître, de se posséder et de librement se donner et entrer en communion avec d'autres personnes, et il est appelé, par grâce, à une alliance avec le Créateur, à lui offrir une réponse de foi et d'amour que nul autre ne peut donner à sa place» (Conseil pontifical, 2005, p. 61-62).

Mais cette prise en compte d'une juste temporalité convoque également les soignants et accompagnants, leur rappelant qu'ils ne possèdent pas le dernier mot de l'histoire du sujet souffrant, que mystérieusement un avenir, un salut est toujours possible puisque "l'heure ne nous est pas connue» (Mt 24,36). Or, bien des situations d'épuisement auraient parfois tendance à vouloir maîtriser ce temps (Jacquemin, 2002, p. 148). Ce risque peut se traduire, comme le souligne B. Cadoré (Cadoré, 2001), dans trois directions au moins. Il pourra tout d'abord s'agir de la personne qui ne supporte plus la durée de ses propres jours, et ce d'autant plus lorsque ces jours ne lui sont plus renvoyés comme possiblement signifiants, dignes par un entourage. Dans pareil contexte, la clôture du temps devient l'unique lieu de sens. Cette difficulté de tenir dans le temps pourra être vécue par le médecin, l'équipe soignante qui ne parviennent plus, dans leur effort thérapeutique, à soutenir l'espoir, «l'à-venir », de la personne souffrante. Cette attitude sera d'autant plus prégnante - nous en avons déjà largement traité que le seul rapport à la maladie et au corps de l'autre souffrant aura été modelé par une vision technique et d'efficacité dans l'exercice médical: «quand on ne sait plus rien faire, quel sens aurait encore l'existence?». Répondre à la mort de l'autre comme abrègement de son temps deviendrait ainsi le dernier lieu de l'efficacité, $\mathrm{du}$ «savoir-faire». Enfin, cette tentation pourra traverser un entourage épuisé par le temps. Ce sont toutes ces situations où l'entourage se trouve submergé par un temps de la maladie ou un temps du mourir auquel, par épuisement dans l'investissement et la durée, ils ne parviennent plus à donner sens; cette posture de l'entourage étant parfois induite par un difficile rapport au pronostic qui, d'une manière indue, a été établi ou compris comme une maîtrise sur le temps: "encore pour autant de jours...». Or, en pareilles circonstances «trois réalités qui caractérisent notre rapport au temps se trouvent ainsi profondément déplacées : l'attachement à la vie du sujet lui-même, le soutien "thérapeutique" de la vie d'autrui contre ce qui l'emporte, le sens de la relation durable avec autrui dans la confrontation au tragique de l'existence» (Cadoré, 2001, p. 3).

Cette difficulté de rencontrer l'autre dans son temps se trouvera généralement renforcée, comme nous l'avons considéré en préalable, par une temporalité sociale qui rend de plus en plus malaisé le temps long de la solidarité au profit d'un temps court qu'elle aurait trop tendance à qualifier de solidaire par l'exécution du seul geste «ponctuel», celui de permettre à l'autre d'en finir avec le temps, avec sa vie.

\section{UNE ANTHROPOLOGIE DU TRAITEMENT \\ DE LA DOULEUR}

Si le respect du temps de l'autre avec ce qu'il implique d'accompagnement est une donnée essentielle, il ne va pas sans un soulagement de la douleur qui mérite également toute sa qualification: mettre en œuvre une thérapie de la douleur invite à réfléchir sur le statut de la personne soignée. En effet, cette importance du traitement de la douleur fut, il

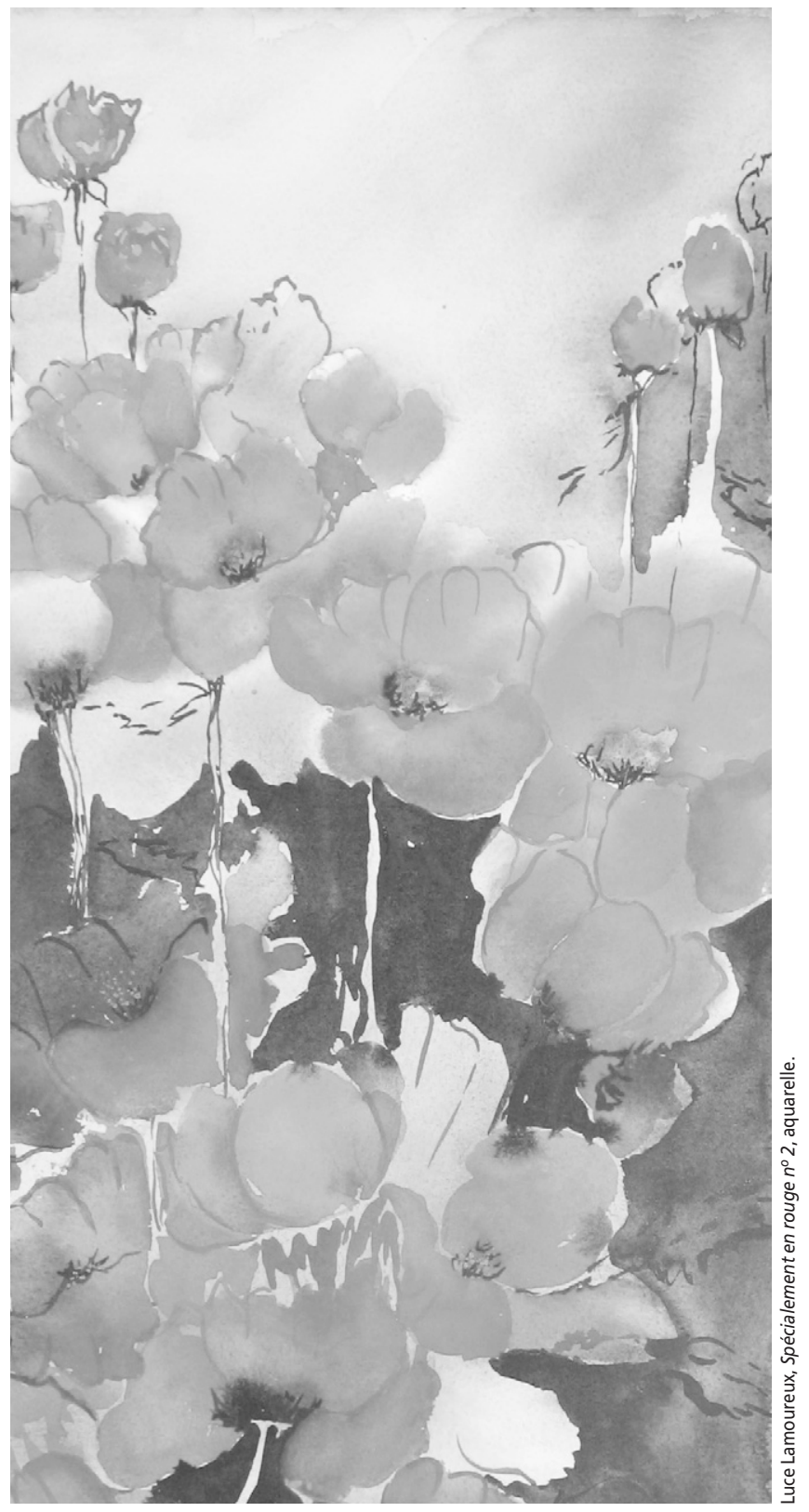
y a plus de cinquante ans, soulignée comme essentielle par l'Église catholique, particulièrement par la voix de Pie XII : «Quels sont les motifs qui permettent dans les cas d'espèce d'éviter la douleur physique sans entrer en conflit avec une obligation grave ou avec l'idéal de la vie chrétienne? On pourrait en énumérer un grand nombre; mais, malgré leur diversité, ils se ramènent finalement au fait, qu'à la longue, la douleur empêche l'obtention de biens et d'intérêts supérieurs. Il peut se faire qu'elle soit préférable pour telle personne déterminée et dans telle situation concrète; mais, en général, les dommages qu'elle provoque forcent les hommes à se défendre contre elle...» (Pie XII, 1957, p. 354).

Mais le caractère impératif d'une juste prise en charge de la douleur apparaît nettement lorsqu'on le met en balance avec deux attitudes encore trop présentes dans la prise en charge de la fin de vie : l'acharcôté comme de l'autre, la question de la douleur se trouve concernée: l'acharnement thérapeutique risque de ne pas prendre en compte le vécu douloureux du patient tandis que l'euthanasie représenterait le passage à l'acte pour y mettre un terme. On peut dire qu'en se situant dans une voie moyenne, les soins palliatifs s'insurgent contre une volonté de maîtrise, d'objectivation de l'autre et du corps de l'autre, d'autant plus que cette prise en charge de la douleur n'est pas simplement assimilable à la prise en compte de la douleur physique mais bien à la prise en compte de ce que nous avons appelé la douleur totale (total pain).

En effet, la tentation est grande parfois de s'en tenir au seul acharnement thérapeutique lorsqu'on se situe dans l'appréhension de la mort proche: une attitude nement thérapeutique et l'euthanasie. D'un 
qui permet de se cantonner dans le seul soin technique, l'objectivisme biologique et fonctionnel, faisant "comme si... », occultant artificiellement la situation réelle du malade en fin de vie. Cette approche uniquement technicienne que refusent les soins palliatifs empêche la rencontre du malade dans ses besoins réels mais offre la sécurité d'un paravent technique. L'autre dimension d'objectivation serait l'euthanasie comme masque mis sur la mort d'autrui, comme passage à l'acte légitimé en tant qu'issue à une situation thérapeutique devenue problématique. Notre but n'est certes pas ici de nous attarder sur ces deux questions éthiques spécifiques mais de souligner que le traitement de la douleur adéquatement conduit permet de ne pas fermer le questionnement relatif à la fin de la vie, dans le chef du malade certainement mais également dans celui des soignants et de toutes les personnes en situation d'accompagnement.

\section{L'ACCOMPAGNEMENT, MANIFESTATION DE LA DIGNITÉ D'AUTRUI}

Cette acceptation d'entrer en relation avec le malade constitue également un enjeu important pour rencontrer le patient dans toute sa dignité d'humain : soignants, famille et accompagnants prennent le risque de se situer sur un terrain d'égalité qu'est celui de l'humanité de l'autre qui me renvoie à ma propre humanité. Il importe de reconnaître que l'autre malade est un humain comme nous, tissé des mêmes besoins, d'attentes relationnelles et de reconnaissance semblables. Cette dimension d'accompagnement atteste implicitement que l'autre, le malade, est encore quelqu'un, une personne avec ses besoins, ses attentes, ses questions. Bien sûr, pour perdurer dans cette dimension éthique du soin, il importe de garder une justesse dans cette relation, de ne pas croire qu'il sera possible de "tout solutionner» chez l'autre ou, plus tragiquement encore, de le conduire sur nos propres chemins, comme s'il existait, à nos yeux, une fin de vie «idéale et à atteindre absolument».

À ce sujet, B. Matray proposait encore deux éléments d'évaluation de la justesse de toute relation d'accompagnement. Tout d'abord, il s'agit de rester sans cesse en éveil dans cette relation afin de percevoir ce que souhaite réellement l'autre à ce moment-ci, à cet instant où nous sommes effectivement en présence de lui. Et cette présence à l'autre ne peut à nos yeux se vivre sans une présence équivalente à nous-mêmes : comment ces valeurs, ces questions, ces images de la personne en fin de vie nous renvoient également à ce que nous vivons, à ce que nous sommes, à nos visées de la vie bonne, à nos valeurs? En fait, nous retrou- vons, au cœur d'une pratique d'accompagnement, cette importance du temps, un temps qui fait entrer conjointement accompagnants et soignés dans l'immédiateté de ce qu'ils sont et vivent. Cette attention au présent de la relation d'accompagnement amène le deuxième élément d'évaluation: "la relation d'accompagnement doit chercher à se libérer de toute violence» (Matray, 1995, p. 10). La violence radicale, dépassant le caractère en soi violent de toute fin de vie, consisterait ici à sans cesse projeter l'immédiat de la relation dans un futur qui est le nôtre : imposer à l'autre nos conceptions de la fin de vie, de la «bonne mort», de la maladie, de la souffrance, etc. Entrer dans ce type de rapport au malade conduirait à l'exproprier lui-même de son propre chemin et empêcherait toute personne d'être en réelle situation d'accompagnement; nous ne serions que dans une situation d'abusive guidance. Accepter ce préalable à tout accompagnement revient à souligner conjointement un relatif désintéressement pour laisser à l'autre toute sa place dans son propre cheminement, lui offrant, comme le soulignent les évêques belges, les moyens de réaliser à son rythme son propre chemin, y compris de faire de sa mort le point culminant de sa vie: «Nous voulons néanmoins attirer l'attention sur le caractère de plénitude que revêt parfois l'adieu conscient et paisible aux choses et aux personnes. Chaque fois que nous en sommes témoins, nous disons que ce fut une belle mort. C'est le moment où l'être humain met la dernière touche, très personnelle, à son existence, et couronne pour ainsi dire l'œuvre de sa vie» (Évêques de Belgique, 1994, p. 236).

\section{UNE CONCEPTION RENOUVELÉE \\ DU TRAVAIL EN ÉQUIPE}

Nous voudrions encore mettre en évidence un autre élément d'évaluation de ce que pourrait signifier la rencontre de l'autre dans sa dignité, dans ce qui le maintient comme sujet: le travail interdisciplinaire en équipe. Il importe d'en relever la nécessité concrète à plusieurs niveaux. Le premier niveau souligne l'importance de garder un équilibre dans l'exercice concret de la fonction soignante: il s'agit tout d'abord de tenir dans cet engagement exigeant qu'est la confrontation perpétuelle avec l'idée et la réalité de la mort; il s'agit aussi de pouvoir dire, partager ce qu'on vit, ses difficultés, ses questions. Mais cette notion d'équipe est d'autant plus importante qu'il s'agit, comme nous l'avons vu, de rencontrer le malade dans l'ensemble de ses besoins: nul ne peut prétendre à l'omnicompétence en matière médicale, sociale, psychologique, spirituelle - et il importe que chaque acteur du soin puisse le reconnaître! Ce n'est qu'en comptant sur l'unité d'engagements diversifiés et coordonnés - dont le malade sera toujours le centre - qu'il est possible de développer une approche holistique des soins. Cette prise en charge interdisciplinaire du malade conduit davantage l'équipe à une structuration moins hiérarchique mais guidée par la reconnaissance des mutuelles compétences en complémentarité. Cette approche nous renvoie assez facilement à l'idéal paulinien de la multiplicité des charismes à exercer pour le bien de tous (1 Co 12,1-11), chacun ayant reçu un don

SOIGNANTS, FAMILLE

ET ACCOMPAGNANTS PRENNENT LE RISQUE DE SE SITUER SUR UN TERRAIN D'ÉGALITÉ QU'EST CELUI DE L'HUMANITÉ DE L'AUTRE QUI ME RENVOIE À MA PROPRE HUMANITÉ.

particulier pour le bien de tous. Cet idéal de l'apôtre pourrait être, pour le croyant, une raison supplémentaire pour considérer dans sa spécificité chaque professionnel comme porteur d'un don particulier et complémentaire pour la prise en charge de la personne malade ou en fin de vie.

Enfin, cette dimension du travail en équipe trouve sa qualification majeure dans le fait qu'elle permet le maintien d'un consensus fondateur: le pari d'être au service d'une personne en situation de fin de vie, dans une optique de qualité de vie. Et ce consensus fondateur révèle la dimension éthique dont l'équipe se trouve porteuse au niveau des valeurs qu'elle met en œuvre dans les soins et l'accompagnement. Or, des situations de crises liées à l'état d'un malade ou à l'équipe elle-même surviennent dans la pratique. C'est en se réappropriant par l'échange, la discussion, les valeurs auxquelles on croit qu'il est également possible de tenir dans l'engagement. En ce sens, l'existence de l'équipe implique une capacité interdisciplinaire d'éthique clinique (Cadoré, 1997) : une capacité de s'interroger sur la signification de sa pratique et de ce qui est décidé dans l'intérêt du malade lui-même. En d'autres mots, c'est dans une visée commune, partagée, que peut également être réappropriée et rencontrée la dignité de la personne souffrante alors que tant de situations de soin non réfléchies en commun, nourries de plusieurs compétences, auraient tendance à l'altérer. 


\section{LE SOIN, ESPACE}

\section{D'UNE RENCONTRE INDIVIDUELLE}

Et ceci nous conduit au dernier élément d'évaluation critique permettant au patient d'être maintenu dans sa dignité de sujet, la place centrale qu'on lui accorde dans les faits et décisions. Il s'agit, selon David J. Roy, d'un des principaux défis des soins palliatifs: "[...] le plus grand défi de l'éthique clinique dans le domaine des soins palliatifs est d'arriver à poser, dans chaque cas, un jugement pratique permettant d'atteindre le consensus nécessaire en vue de répondre dans la plus grande mesure du possible aux besoins, désirs et plan de vie du patient» (Roy, 1992, p. 173). Fondés sur l'approche holistique du patient dans la rencontre de ses besoins par une équipe interdisciplinaire, l'idéal des soins palliatifs rappelle certes l'importance de placer le patient au centre de la pratique et de la réflexion. Cependant, ce recentrage sur la personne du patient ne pourra se faire que moyennant le développement d'une interrogation critique sur le sens du soin, moyennant une interrogation sur la dimension éthique de ce dernier, autrement dit une interrogation sur le sens de l'action: une capacité apprise de rendre compte mutuellement et en raison de ce qui motive nos choix thérapeutiques et d'accompagner la personne en fin de vie. Il s'agit de réapprendre sans cesse, et en équipe, la capacité du patient d'être sujet de soin dans la totalité de son existence corps et histoire - comme la norme ultime qui va régir la moralité des décisions et actes médicaux.

Cette approche unique du patient par le regard pluriel et concerté de l'ensemble des soignants - révèle un des enjeux éthiques majeurs du soin et de la rencontre du patient devenu en Christ, tout comme nous, fils de Dieu, capable tout comme nous de participer à la vie même de Dieu (Ep 1,4-5). En ce sens, reconnaître le patient comme sujet singulier permettrait de nommer théologiquement le concept de dignité en y reconnaissant et attestant, par une pratique professionnelle, la présence de Dieu: "En vérité, la dignité n'est rien d'autre que la marque de la Présence divine qui transfigure la liberté de la personne et lui permet de poser des actes toujours plus conformes à l'Évangile» (Thévenot, 2000, p. 212).

\section{MOURIR DANS LA DIGNITÉ, UN LIEU POUR DIEU?}

Après nous être efforcé de montrer en quoi et comment la dignité pouvait être considérée comme un concept en actes dans la relation de soins, nous aimerions, pour terminer, ébaucher certaines réflexions de nature plus théologique pour

laisser résonner la notion de dignité en ce lieu. En effet, il importe de pouvoir se demander ce que le recours à la notion de dignité peut signifier pour le croyant, que ce soit en termes de points d'appui ou de contestation de sa foi. De plus, pour

C'EST DANS UNE VISÉE COMMUNE, PARTAGÉE, QUE PEUT ÉGALEMENT ÊTRE RÉAPPROPRIÉE ET RENCONTRÉE LA DIGNITÉ DE LA PERSONNE SOUFFRANTE ALORS QUE TANT DE SITUATIONS DE SOIN NON RÉFLÉCHIES EN COMMUN, NOURRIES DE PLUSIEURS COMPÉTENCES, AURAIENT TENDANCE À L'ALTÉRER.

le croyant, parler de l'homme peut-il se faire sans parler de Dieu, y compris lorsque la figure de l'homme se trouve altérée? Enfin, le recours au discours théologique devrait permettre d'ouvrir, comme l'avait fait la deuxième section de cet article, la question des comportements : le recours à la théologie peut-il être source d'un éclairage complémentaire dans l'évaluation morale de l'action humaine?

Ainsi, dans un premier temps, nous nous demanderons si la dignité de l'humain mourant peut être qualifiée comme un lieu de Dieu et pour Dieu. Nous aimerions montrer ensuite comment la juste présence humaine et soignante telle que nous l'avons évoquée pourrait, elle aussi, devenir un lieu d'attestation de non étrangeté de Dieu à ce que vit la personne au terme de son existence. Ceci devrait nous permettre de pouvoir nommer cet engagement comme un lieu ecclésial attestant de Dieu et donnant un certain visage à l'Église de ce temps.

\section{MOURIR, UNE EXPÉRIENCE}

DIGNE DE DIEU

Dans un premier temps, nous voudrions nous arrêter à deux événements de la foi chrétienne qui sont suffisamment essentiels, marquants pour indiquer que l'expérience humaine du mourir en sa dignité serait également une expérience qui dit la dignité, la grandeur de notre Dieu. Il existe certes une multitude de passages évangéliques où cette dimension est présente mais nous nous arrêterons uniquement à l'événement mort-résurrection ainsi qu'à l'épisode de la transfiguration pour essayer de dire l'«en-soi du sens » du mourir et de son accompagnement pour le croyant.

\section{L'événement mort-résurrection}

Pour le croyant, l'événement passion, mort, résurrection (Jacquemin, 2002, p. 112-120) constitue certainement le moment de la Révélation qui dessine, par excellence, non seulement jusqu'où peut aller l'amour du Christ pour l'humain mais conjointement, et d'une manière profondément indissociable, quel est ce Dieu humanité, le souci, le projet pour l'ensemble de cette dernière. Comme le souligne A. Gesché (Gesché, 2001, p. 139), le mot «résurrection» renvoie certes à l'image d'une remise debout mais atteste surtout du lieu même d'origine de ce relèvement du Christ qui trouve, pour le croyant, tout son sens: car c'est quelqu'un qui, passé par la mort de la croix et le séjour au tombeau, est remis debout, dans son état initial pourrions-nous dire, celui de Fils de Dieu. Jésus le Serviteur souffrant, rejeté et humilié est bien celui qui se trouve redressé et sauvé d'un abaissement accablant (Gesché, 2001, p. 141). Pour le croyant, ce passage de Jésus par la mort et son relèvement par et en Dieu constitue un acte transcendant par excellence: relevé des morts, Jésus passe auprès du Père, est établi par lui et par la puissance de l'Esprit «Seigneur des vivants et des morts» (Ac 5,31 s.).

Pour saisir l'ampleur de cette affirmation, et par voie de conséquence ce qu'elle peut signifier pour la foi des croyants, il importe de bien comprendre que la résurrection du Christ et le salut qui en résulte tant pour lui que pour tout homme ont un lieu qui est loin d'être innocent puisqu'il s'agit bel et bien du séjour des morts (Gesché, 2001, p. 171-172) d'où le Christ est relevé, devenant pour l'humain source de salut. Nous pouvons ainsi comprendre que la résurrection représente une victoire non sur une simple mort, mais bien sur toute mort car c'est bien en son domaine, en son lieu propre qu'elle se trouve vaincue par le Christ: elle ne peut le retenir, il en est re-surgi par le Père, elle devient le lieu d'un réveil possible pour tout homme. Et c'est là la portée anthropologique essentielle de la résurrection pour le croyant: plus rien, même pas la mort n'est capable de séparer l'homme de Dieu. «La nouvelle formidable de la Résurrection consiste à dire 
que l'homme est sauvé de cette situation, qu'il ne vivra pas séparé de Dieu » (Gesché, 2001, p. 176).

Cet événement de la résurrection, cette re-suscitation de Jésus par le Père, fut retranscrite par les Évangélistes avec certains accents qui méritent qu'on s'y arrête, plus particulièrement au regard de ce que peuvent vivre des personnes en situation d'échec. La Résurrection du Christ va de pair avec une promesse, celle d'une présence permanente de Jésus auprès des siens: «Et moi, je suis avec vous tous les jours, jusqu'à la fin des temps» (Mt 28,20). Cette promesse d'une présence indique très clairement que ni la souffrance, ni le trépas ayant conduit à l'ensevelissement, ne pourra faire obstacle dans la vie de chaque homme ou femme et ne pourra être appréhendé comme un lieu indigne de l'homme.

En ce sens, l'affirmation de la résurrection de Jésus, comme passage par la souffrance de la croix, mort et relèvement en Dieu et par Dieu, si elle met au jour des données théologiques essentielles pour dire à la fois qui est Dieu et qui est son Christ, se trouve en même temps porteuse de nombreuses implications existentielles attestant à la fois d'une dignité de l'homme dans sa mort puisque cette dernière est également un lieu où Dieu se dit et se donne. Tout d'abord, et ici encore A. Gesché le dit admirablement (Gesché, 2001, p. 35), la résurrection du Christ réaffirme une non-étrangeté essentielle de Dieu au drame de l'existence, y compris dans les dimensions les plus recluses que sont ces passages par la souffrance et la mort. En ce sens, les hommes et les femmes confrontés à des décisions de l'ordre de la «passion» (comme souffrance et passage) devraient pouvoir s'expérimenter en situation de non-étrangeté à l'égard de Dieu.

Mais sans doute, est-ce encore bien davantage le visage d'un Dieu capable d'accompagner l'humain qui se révèle dans l'existence du Christ, dans son passage par la mort et la résurrection: «Un Dieu qui accompagne, en vertu même de sa nature, non en contradiction avec celle-ci, dans notre histoire faite, elle aussi, de passions, dans tous les sens du terme, celles de la souffrance et celles de l'amour, et que l'on retrouve, à nous définitivement fraternelle et divine, dans la Passion, dans la Compassion du Christ» (Gesché, 2001, p. 39). Ainsi, face aux situations joyeuses de l'existence - où, il faut le reconnaître, Dieu semble nettement moins requis par l'homme contemporain - et aux drames qui l'accompagnent, il est possible à l'humain de ne plus supporter «ce Dieu impassible de l'histoire » mais de le retrouver présent, le précédant au cœur de son quotidien. Par Jésus, Dieu est devenu, pour toujours et pour tout homme, un témoin actif de chaque histoire humaine lui donnant toute sa dignité.

En ce sens, «mourir dans la dignité» peut effectivement être, en l'humain, un lieu pour et de Dieu: l'être de l'homme n'est pas d'être voué ni à la solitude, ni à l'étrangeté à l'égard de ce Dieu. En effet, lorsque l'homme se sent seul, abandonné, indigne, il serait possible d'affirmer qu'il s'agit, au-delà de l'impression subjective et d'un vécu parfois bien réels, d'une erreur d'interprétation théologique: un Dieu est toujours disponible, le précédant même, ayant lui-même, en Jésus, assumé le poids et les conséquences de la souffrance, de son passage et de la mort.

\section{L'épisode de la transfiguration}

Après cet événement central de la mortrésurrection, nous aimerions nous arrêter à un autre épisode de l'Évangile, celui de la transfiguration qui, lui aussi à sa manière, atteste qu'à travers l'altération, ce qui, dans son extériorité, est laid, indigne au sens trop contemporain du terme, peut se manifester la gloire de Dieu. Cette approche nous semble d'autant plus importante qu'elle peut être signifiante pour tous les soignants ayant à prendre soin du corps mais également pour tant de nos contemporains séduits, comme nous l'avons vu, par le corps étincelant qui, seul, pourrait attester de la grandeur de l'humain.

Comme le dit Philippe Lefèbvre, «la transfiguration est une expérience courante pour ceux qui savent voir » (Lefèbvre, 2006, p. 1). En effet, qui de nous n'a pu découvrir dans un corps souffrant l'intensité de certaines vies? Qui n'a pas vu surgir une joie réelle dans un visage fatigué lors d'une visite? Qui n'a pu découvrir tous les traits d'une vie dense et belle dans le corps fatigué d'une personne très âgée? Ces quelques expériences seraient de nature à nous faire percevoir qu'un certain regard porté sur l'autre souffrant permettrait de découvrir que la vie est toujours plus que ce qui s'en donne à voir dans l'immédiateté: "C'est que la chair dont nous sommes faits parle; la chair témoigne que la vie qu'elle a accueillie se trouve bien en elle, mais que cette vie ne vient pas d'elle. La vie vient de plus loin que nous: ce qu'il y a de beau dans certains corps flétris, flageolants, c'est cette évidence de la vie alors que les apparences disent l'usure, la dégradation. La chair incapable de produire la vie est pourtant habitée par la vie, révélée par la vie» (Lefèbvre, 2006, p. 1).

C'est bien à cette expérience que Jésus convie ses disciples lors de la transfiguration (Mc 9,2-10) : percevoir dans un corps connu une lumière, une vie qui vient d'ailleurs, une lumière qui envahit la tota- lité du Christ, son corps, ses vêtements; même l'espace s'en trouve transfiguré, et tout cela devient source de joie pour Pierre, Jacques et Jean: «Rabbi, il est bon que nous soyons ici» (Mc 9,5). Cette joie des disciples consistant à faire l'expérience d'une rencontre de Dieu, un Dieu qui se donne à reconnaître dans le quotidien du corps, est devenue possible moyennant l'acceptation d'ouvrir un autre regard, celui qui n'est pas de l'ordre de l'immédiateté de la perception, ni celui auquel le monde nous convie spontanément, mais bien celui de «l'écart» (Mc 9,2) où le Christ aime nous conduire.

Si cet épisode a comme visée pédagogique d'aider les disciples à appréhender le possible de la résurrection, il nous redit une fois de plus le caractère central de cette dernière où, comme le dit $\mathrm{M}$. Bellet, le Christ peut devenir l'un de nous, nous ouvrir à la promesse d'une vie à travers tout ce qui, a priori, dirait l'indignité de l'humain: «Or lui, que tout désigne comme le juste, le sage, le prophète et même le roi, il descend dans l'en-bas, jusqu'au monstrueux avilissement, bafoué, couvert de crachats, couronné d'épines, crucifié entre deux bandits. Il devient l'un deux. En sorte qu'ils peuvent dire, celles et ceux d'en bas, ils peuvent dire en vérité: "Il est des nôtres"»(Bellet, 2005, p. 111). Dans notre épisode, le corps altéré, fatigué - il avait bien fallu monter sur la montagne en pleine chaleur - peut être reconnu comme un corps porteur d'une autre lumière, d'une autre vie.

C'est en ce sens que l'épisode de la transfiguration nous paraît fondamental pour la question relative à la dignité $d u$ mourir. L'expérience de la transfiguration du Christ est, pour le croyant, une invitation éthique à reconnaître tous les êtres transfigurés d'aujourd'hui, à aller au-delà des apparences, pour autant que nous puissions accueillir une vie d'ailleurs, celle que l'Esprit nous donne de pouvoir découvrir - "Celui-ci est mon Fils bien aimé» (Mc 9,1) - à travers tout humain. En fait, lors de la transfiguration, Jésus éduque ses disciples, et donc nous-mêmes, à porter sur tout homme le regard même de Dieu, car en tout être humain fils de Dieu, il y a la gloire de Dieu à décrypter et à accueillir. Bien sûr, il s'agit ici d'une visée de la foi et les croyants n'ont pas à imposer ce regard, cette vision de la réalité - Jésus recommande d'ailleurs aux siens de ne pas parler trop vite (Mc 9,9) - mais ce regard porté sur l'autre souffrant devrait conduire les croyants à ce que nous nommerions une éthique de la transfiguration donnant tout son poids au concept clinique de dignité. Certes, ce regard présuppose un engagement, celui du regard évangélique: 
tout homme, toute femme est sans cesse bien plus que ce qu'il n'en donne à voir. Et, comme nous l'avons vu, c'est dans l'engagement soignant, la présence au quotidien que cette conviction de foi pourra transparaître dans le respect conféré au plus souffrant.

\section{UN ENGAGEMENT HUMAIN}

ET PROFESSIONNEL QUI DIT DIEU

On a vu précédemment comment la dignité du malade mourant, si elle avait de nos jours certaines difficultés à être reconnue en tant que dimension constitutive, inaliénable de l'humain, pouvait

\section{UN CERTAIN REGARD PORTÉ}

\section{SUR L'AUTRE SOUFFRANT}

PERMETTRAIT DE DÉCOUVRIR

\section{QUE LA VIE EST TOUJOURS PLUS}

\section{QUE CE QUI S'EN DONNE À VOIR}

DANS L'IMMÉDIATETÉ.

cependant être attestée, révélée dans sa dimension clinique par l'engagement de tant de professionnels, familiers et accompagnants dans leur diversité. La dignité de la personne en fin de vie est donc un fait à nos yeux et une approche théologique ne peut qu'y insister davantage lorsqu'elle nous indique que cette manière de la rencontrer engage non seulement l'agir même du Christ (Lc 10,25-37) mais surtout la manière concrète, pour le croyant, de le rencontrer (Mt 25,31-46).

\section{La parabole du Bon Samaritain}

\section{(LC 10,25-37)}

Cette parabole est suffisamment connue de tous pour qu'on s'y arrête trop longuement mais elle reste très significative pour notre sujet. Elle l'est tout d'abord au niveau de son enjeu central, la question du salut: «Maître, que dois-je faire pour avoir en partage la vie éternelle?»(v. 25). Au-delà de la seule adéquation à la loi, ce sera un comportement concret qui sera proposé par le Christ, semblable au sien: "Va, et toi aussi fais de même» (v. 37). En effet, si cette parabole nous indique d'abord quel est le comportement du Christ, révélateur de l'agir de Dieu, c'est bien lui le premier samaritain, témoin d'un Dieu proche, qui se laisse toucher par celui, celle qui est laissé de côté, sur le bord du chemin; ce que pourrait être, dans un certain fonctionnement de la médecine, la personne en fin de vie.
Mais, par voie de conséquence, c'est surtout l'agir du disciple, du chrétien qui se dessine dans ce même récit. Le salut chrétien se joue, d'une certaine manière, dans cette capacité à se faire proche de celui qui, comme un anonyme, un exclu tenu à distance, et de le restaurer dans son humanité même lorsqu'il est roué de coups, à moitié mort (v. 30) et qu'il n'a plus visage humain (Is 52,14). C'est dans cette rencontre du blessé, bien au-delà des seuls pansements déposés sur ses plaies, que se dit la dignité de l'humain, dignité attestée par le Christ lui-même dans un comportement signifiant le salut: «[...] le juste respect de la dignité de celui qui est affronté à la mort consiste à l'aider à reconstituer des liens profondément humains, là où le mal tend à créer de l'isolement, du morcellement et, pour tout dire, une certaine perte d'identité» (Thévenot, 2000, p. 216). Ceci nous renvoie pleinement à la parabole où le blessé n'était plus personne - certains se refusent à le voir (v. 30 et 32 ) - et où seul un geste d'hospitalité le conduisant à l'auberge lui donne d'être accueilli et restauré comme humain (v. 34): c'est en se faisant proche du blessé que le disciple, à l'image de l'engagement de Dieu, lui dira qui il est.

Lorsque nous agissons de la sorte en reconnaissant l'homme digne dans le défiguré, en y découvrant un comportement où se joue le salut, nous agissons certes à l'image du Christ, premier samaritain, mais nous attestons, au cœur d'une pratique humaine et professionnelle de qui est notre Dieu, un Dieu proche et dont seule la notion d'excès peut dire combien le plus souffrant compte pour lui. N'est-il pas ce Dieu qui, ayant tout fait, s'assure encore de venir rencontrer le manque (v. 35), ce qui dans l'immédiateté du soin ne se donnait pas à panser?

\section{Le jugement dernier (Mt 25,31-46)}

Le texte du jugement dernier permet de faire un pas supplémentaire dans cette insistance à faire de la dignité de l'autre souffrant un lieu de la rencontre inter-humaine disant qui est notre Dieu. En effet, lors du jugement, l'enjeu de la vie chrétienne se trouve nettement signifié par la capacité à se faire proche du plus fragilisé dans l'existence, quelle qu'en soit la cause. Tout ce qui est fait ou non fait en ce sens se trouve clairement assimilé à un acte posé ou non en faveur du Christ. Il est même intéressant de noter la surprise de certains témoins de ce discours - «mais quand avons-nous fait ou pas fait cela?» (v. 37) - indiquant que c'est bien le comportement quotidien qui, pour le croyant, est le lieu de la rencontre du Christ. Et pourtant, lorsque le plus fragile se trouve reconnu comme un semblable digne d'attention, c'est la bonne nouvelle du Royaume qui se dit et se réalise (Lc 4,18-21).

Mais le texte va, nous semble-t-il, encore plus loin lorsque le Christ pose une équivalence entre sa personne et le plus fragilisé puisque c'est bien lui, Jésus, qui aura été objet ou non d'attention, de sollicitude. Cette dimension est en mesure de nous faire appréhender combien cette notion de dignité de l'humain est, pour le croyant, foncièrement inscrite dans un au-delà des apparences puisque rencontrer l'autre fragile, souffrant, conduit à rencontrer le visage même du Christ. Ceci rejoint pleinement la profondeur donnée à cette valeur dans le discours contemporain de l'Église: «Étant donné que sur le visage de tout homme resplendit quelque chose de la gloire de Dieu, la dignité de chaque homme devant Dieu constitue le fondement de la dignité de l'homme devant les autres hommes» (Conseil pontifical, 2005, p. 79).

\section{OUVERTURE ECCLÉSIALE ET PASTORALE:} UNE IMAGE DE DIEU EN ACTES

Pour conclure ce parcours où nous nous sommes efforcé de proposer l'urgence d'une reconnaissance de la dignité de l'homme au cœur de l'advenue de sa propre mort, d'en indiquer certes une présence en soi, inaliénable mais ayant surtout à se manifester dans des comportements humains et professionnels qui, pour le croyant, iraient jusqu'à engager Dieu et le salut, nous aimerions envisager une ultime question: que nous dirait de l'Église comme communauté de croyants semblable comportement?

Nous voudrions partir ici du texte de Paul (1 Co 12,12-14) où il est redit que l'Église est à l'image d'un corps dont tous les membres, inscrits en Christ, occupent une place particulière pour le bien de l'ensemble. Comme le dit A. Join-Lambert, le tout n'est pas de faire dire à ce texte ce qu'il ne dit pas «ni d'en faire par anachronisme une recette de comportement pour aujourd'hui » (Join-Lambert, 2006), mais de mettre au jour combien, par son comportement, le chrétien peut avoir de nos jours une mission prophétique, engageant l'Église qu'il est, particulièrement au regard de la question qui nous occupe. Ce passage paulinien nous rappelle que le chrétien, membre du corps du Christ qu'est l'Église, partage avec chacun une égale dignité et qu'il atteste de la beauté de ce corps lorsqu'il témoigne de cette même dignité pour chaque membre du corps, participant de la sorte au ministère même du Christ: "Toute la communauté des croyants est, comme telle, sacerdotale. Les fidèles exercent leur sacerdoce baptismal à travers leur participation, chacun selon 
sa vocation propre, à la mission du Christ, Prêtre, Prophète et Roi » (Catéchisme, 1992, p. 330).

Or, au regard de tous les points d'attention clinique révélateurs de la dignité du plus souffrant que nous avons pu décliner en deuxième partie, le professionnel atteste, même sans le vouloir ni le savoir (Mt 25,37), qui est ce corps dont il fait partie, de qui est cette Église dont il est membre. Nous l'avions déjà signifié en introduction en redisant la pleine équivalence entre les soucis de l'humain aujourd'hui et les soucis de l'Église, le parcours ici réalisé nous indique que la rencontre de l'humain en sa dignité dit qui est l'Église: "L'humain est le chemin de l'Église puisque le Christ est uni en tout homme» (Jean-Paul II, 1979, p. 54). De plus, cette qualification de l'humain est en mesure de qualifier, pour le croyant, l'action humaine qui va à la rencontre de l'autre humain: elle n'est pas simplement anthropologique, sociale mais devient spécifiquement théologique et ecclésiale puisque la réalité du monde devient le lieu même où l'action de l'homme ordonne le projet même de Dieu (Jean-Paul II, 1987, p. 37).

Et nous donnerions volontiers le mot de la fin à Benoît XVI lorsqu'il signale combien, pour le professionnel soignant, s'impose une nécessaire compétence technique, scientifique à travers laquelle pourra transparaître, pour le plus souffrant, un signe de sa foi attestant du Dieu d'amour:

En ce qui concerne le service des personnes qui souffrent, la compétence professionnelle est avant tout nécessaire: les soignants doivent être formés de manière à pouvoir accomplir le geste juste au moment juste, prenant aussi l'engagement de poursuivre les soins. La compétence professionnelle est une des premières nécessités fondamentales, mais à elle seule, elle ne peut suffire. En réalité, il s'agit d'êtres humains, et les êtres humains ont toujours besoin de quelque chose de plus que de soins techniquement corrects. Ils ont besoin d'humanité. Ils ont besoin d'attention du cœur. Les personnes qui œuvrent dans les Institutions caritatives de l'Église doivent se distinguer par le fait qu'elles ne se contentent pas d'exécuter avec dextérité le geste qui convient sur le moment, mais qu'elles se consacrent à autrui avec des attentions qui viennent du cœur, de manière à ce qu'autrui puisse éprouver leur richesse d'humanité. C'est pourquoi, en plus de la préparation professionnelle, il est nécessaire pour ces personnes d'avoir aussi et surtout une «formation du cœur»: il convient de les conduire à la rencontre de Dieu dans le Christ, qui suscite en eux l'amour et qui ouvre leur esprit à autrui, en sorte que leur amour du prochain ne soit plus imposé pour ainsi dire de l'extérieur, mais qu'il soit une conséquence découlant de leur foi qui devient agissante dans l'amour (voir Ga 5,6) (Benoît XVI, 2006, p. 62-63).

\section{Bibliographie}

AMEISEN, J.-CL., D. HERVIEU-LÉGER et E. HIRSCH (2003). Qu'est-ce que mourir?, Paris, Éditions Le Pommier.

BELLET, M. (2005). La traversée de l'en-bas, Paris, Bayard.

BENOÎT XVI (2006). Dieu est amour, Paris, Bayard / Cerf / Fleurus-Mame.

CADORÉ, B. (1997). L'éthique clinique comme philosophie contextuelle, Québec, Fides.

CADORÉ, B. (2001). L'ultime requête, Texte CEM/Lille.

COLLECTIF (1992). Catéchisme de l'Église catholique, Paris, Mame/Plon.

COLLECTIF (2000). «Dignité», dans Médecine de l'homme, $\mathrm{n}^{\circ} 245$, janvier-février, p. 5.

CONCILE VATICAN II (1967). Constitution pastorale Gaudium et Spes, n ${ }^{\circ}$ 1, Montréal/ Paris, Fides.

CONSEIL NATIONAL DE L'ORDRE DES MÉDECINS (1995). Code de déontologie médicale, Bruxelles, $47 \mathrm{p}$.

CONSEIL PONTIFICAL JUSTICE ET PAIX (2005). Compendium de la doctrine sociale de l'Église, Namur, Fidélité.

ECHARD, B. (2006). Souffrance spirituelle du patient en fin de vie, Ramonville-SaintAgne, Éditions Erès.

ÉVÊQUES DE BELGIQUE (1994). «Déclaration des évêques de Belgique: l'accompagnement des malades à l'approche de la mort», Documentation Catholique, $\mathrm{n}^{\circ}$ 2089, p. 233.

JACQUEMIN, D. (2002a). Bioéthique, médecine et souffrance. Jalons pour une théologie de l'échec, Québec, Mediaspaul.

JACQUEMIN, D. (2002b). «Les soins palliatifs: enjeux éthiques d'une rencontre. Les soignants face à la personne en fin de vie ", Revue Française des Affaires Sociales, $n^{\circ} 3$, p. $148-151$.

JACQUEMIN, D. (2004a). Éthique des soins palliatifs, Paris, Dunod.

JACQUEMIN, D. (2004b). «Traitement de la douleur du patient en fin de vie: d'une exigence thérapeutique à une affirmation anthropologique", dans Actes de la Journée régionale Prise en charge de la douleur du patient en fin de vie, Lille, Faculté de médecine Henri-Warembourg, Université de Lille II, 24 novembre, p. 37-39.
JEAN-PAUL II (1979). Lettre encyclique Redemptor hominis, Paris, Le Centurion.

JEAN-PAUL II (1987). Exhortation apostolique post-synodale de Jean-Paul II sur la vocation et la mission des laïcs dans l'Église, Paris, Mediaspaul.

JOIN-LAMBERT, A. (2006). Quels enjeux ecclésiaux à l'identité? Quelle présence d'Église?, texte remis au Certificat universitaire de formation théologique "Ethique et pratiques de santé», Faculté de théologie UCL/Cliniques universitaires UCL de MontGodinne, 12 septembre 2006, 4 p.

JUVIN, H. (2005). L'avènement du corps, Paris, Gallimard.

LEFEBVRE, PH. (2006). «Homélie prononcée le dimanche 6 août 2006 ", Supplément au bulletin JDS, $\mathrm{n}^{\circ} 135,45$ bis rue de la Glacière, 75013 Paris.

MARIN, I. (1991). "La dignité humaine, un consens? », Esprit, n ${ }^{\circ}$ 2, p. 97-101.

MATRAY, B. (1995). «Les soins palliatifs: approche éthique», Laennec, Paris, octobre, p. 7 .

PIE XII (1957). «Problèmes religieux et moraux de l'analgésie», dans P. VERSPIEREN (1987), Biologie, médecine et éthique, Paris, Le Centurion, p. 354.

RADCLIFFE, T. (2005). Pourquoi donc être chrétien, Paris, Cerf.

ROY, DAVID J. (1992). "Soins palliatifs et éthique clinique », dans Les Annales de soins palliatifs : les défis, Montréal, Centre de bioéthique, Institut de recherches cliniques de Montréal.

THÉVENOT, X. (2000). «Dignité et fin de vie. À l'occasion d'un Rapport du Comité national d'éthique » dans Etudes, Tome 393, $\mathrm{n}^{\mathrm{o}} 3$, p. $211 \mathrm{~s}$.

\section{Notes}

1. «Les joies et les espoirs, les tristesses et les angoisses des hommes de ce temps, des pauvres surtout et de tous ceux qui souffrent, sont aussi les joies et les espoirs, les tristesses et les angoisses des disciples du Christ, et il n'est rien de vraiment humain qui ne trouve écho dans leur cœur. » Concile Vatican II, Constitution pastorale Gaudium et Spes, $\mathrm{n}^{\circ} 1$, Montréal/Paris, Fides, 1967, p. 173.

2. Nous pensons ici à deux lois récentes en France: Loi du 4 mars 2002 relative «aux droits du patient et à la qualité du système de santé »; Loi Léonetti du 22 avril 2005 relative «aux droits des malades et à la fin de vie».

3. Bien des éléments du Code de déontologie médicale attestent de cette dignité inaliénable due à la personne malade : respect absolu de la vie finissante (art. 95), capacité de mettre fin à des traitements disproportionnés (art. 98), «lorsque le malade se trouve dans la phase terminale de sa vie tout en ayant gardé un certain état de conscience, le médecin lui doit toute assistance morale et médicale pour soulager ses souffrances morales et physiques et préserver sa dignité » (art. 96). (Conseil national de l'Ordre des médecins, 1995.) 